BMJ Open Sport \& Exercise Medicine

\title{
Cryotherapy or gradual reloading exercises in acute presentations of rotator cuff tendinopathy: a randomised controlled trial
}

\author{
Frédérique Dupuis, ${ }^{1,2}$ Eva Barrett, ${ }^{3}$ Marc-Olivier Dubé, ${ }^{1,2}$ Karen M McCreesh,, 4
} Jeremy S Lewis, ${ }^{6,7}$ Jean-Sébastien Roy ${ }^{1,2}$

To cite: Dupuis F, Barrett E, Dubé M-0, et al. Cryotherapy or gradual reloading exercises in acute presentations of rotator cuff tendinopathy: a randomised controlled trial. BMJ Open Sport \& Exercise Medicine 2018;4:e000477. doi:10.1136/ bmjsem-2018-000477

- Additional material is published online only. To view please visit the journal online (http://dx.doi.org/10.1136/ bmjsem-2018-000477).

Accepted 3 December 2018

Check for updates

\section{(C) Author(s) (or their} employer(s)) 2018. Re-use permitted under CC BY-NC. No commercial re-use. See rights and permissions. Published by BMJ.

For numbered affiliations see end of article.

\section{Correspondence to} Dr Jean-Sébastien Roy; jeansebastien.roy@rea.ulaval.ca

\section{ABSTRACT}

Objectives Rotator cuff tendinopathies are the most common shoulder disorders. As persistent symptoms lasting more than 3 months have been shown to be a strong indicator of poor outcomes, it is important to have successful interventions in the acute stage. However, there is no evidence yet to guide clinical interventions in an acute pain context. The objective of this study was to compare the short-term effect of a 2-week gradual reloading exercises programme with the use of cryotherapy on symptoms and function for acute rotator cuff tendinopathy.

Methods This simple-blind, randomised controlled trial included 44 participants with acute rotator cuff tendinopathy who were randomly allocated to either the exercises or cryotherapy group. Symptoms and functional limitations were evaluated at weeks 0,2 and 6 using selfreported questionnaires (Disabilities of the Arm, Shoulder and Hand, Western Ontario Rotator Cuff, and Brief Pain Inventory), while acromiohumeral distance, shoulder strength and active range of motion were evaluated at weeks 0 and 2.

Results Following interventions, both groups showed statistically significant improvements on symptoms and function at week 2 and week 6 compared with baseline. However, there was no significant group $\times$ time interaction. There was no time effect on acromiohumeral distance, shoulder strength and active range of motion, as well as no time $\times$ group interaction.

Conclusion The results showed a statistically significant improvement in symptoms and function in both groups, but there was no difference between the short-term effect of a cryotherapy based-approach and a gradual reloading exercises programme.

Trial registration number NCT02813304.

\section{INTRODUCTION}

Rotator cuff (RC) tendinopathy is considered to be a common source of shoulder pain, ${ }^{12}$ accounting for $50 \%-85 \%$ of shoulder diagnoses. ${ }^{3}$ While the pathoaetiology of RC tendinopathy is still unclear, it is described as the clinical presentation of pain and loss of function. ${ }^{4}$ Excessive tendon loading

\section{What are the new findings?}

- Cryotherapy and a gradual reloading exercise programme are equally effective in the short term to improve functional level and decrease the symptoms in individuals with acute rotator cuff tendinopathy.

- No side effects or increase in symptoms was associated with the execution of gradual reloading exercises in the acute phase of rotator cuff tendinopathies.

How might it impact on clinical practice in the future?

Sports medicine practitioners can consider using either one of these interventions in the acute phase of rotator cuff tendinopathies.

- Cryotherapy and a gradual reloading exercises can be considered for their individual benefits as judged necessary.

is considered a key mechanism of RC tendinopathy, ${ }^{6} 7$ although tendon underloading, ${ }^{8} 9$ age, $^{10}$ genetics $^{11}$ and lifestyle ${ }^{12}$ may also be factors in the pathoaetiology. The stage of RC tendinopathy may influence the underlying pathological processes, with suggestions that an early inflammatory component dominates the early stage and degeneration dominates the later stage. ${ }^{13} 14$

The overall prognosis of RC tendinopathy is highly variable, with $40 \%-50 \%$ of patients reporting persistent pain and disability 6-12 months after seeking primary care consultation. ${ }^{15}$ This highlights the need to deliver effective interventions in the acute stage of RC tendinopathy. Currently, there is a lack of evidence regarding the most appropriate treatment for acute RC tendinopathy.

Treatment options for acute RC tendinopathy include patient education, relative rest, cryotherapy and gradual reloading beginning with isometric exercises. The proposed aim 
of cryotherapy is to reduce pain and minimise swelling of the subacromial structures, thereby increasing the acromiohumeral distance (AHD). However, there is little empirical evidence to support the effectiveness of cryotherapy in the acute stages of RC tendinopathy. An alternative option may be to commence gradual reloading early in the acute stage of RC tendinopathy to restore the tendons' capacity to sustain load and to prevent inhibition of the RC tendons. ${ }^{16}$ Gradual reloading has been shown to be effective for chronic RC tendinopathy. ${ }^{17}$ Such an intervention, however, has never been fully evaluated in a randomised controlled trial (RCT) for acute presentation.

A recent pilot study ${ }^{18}$ demonstrated that people with acute and subacute RC tendinopathy $(<12$ weeks $)$ who received either cryotherapy $(\mathrm{n}=6)$, isometric exercises $(n=7)$ or a combination of both $(n=7)$ achieved reductions in pain and disability after 1 week, with no significant differences in outcomes between groups. However, a fully powered and assessor-blinded RCT is needed to build on these results. Therefore, the following were the research questions of this study:

1. Is a rehabilitation programme containing gradual reloading exercises more effective than a rehabilitation programme containing rest and cryotherapy for reducing pain and disability in people with acute $\mathrm{RC}$ tendinopathy?

2. What effects do these interventions have on shoulder strength, shoulder range of motion (ROM) and AHD?

Our hypothesis is that, in the short term, a rehabilitation programme containing gradual reloading exercises will be more effective in reducing pain and disability level as it may prevent RC tendon inhibition and rehabilitate load tolerance.

\section{METHODS}

\section{Participants, therapists and centres}

Adults (aged between 18 and 65 years) with acute $(<6$ weeks) unilateral symptomatic RC tendinopathy who presented a positive sign in each of the following categories were recruited: (1) painful arc of movement; (2) positive Neer or Kennedy-Hawkins tests; and (3) pain on resisted isometric lateral rotation or abduction, or positive Jobe test. The diagnosis accuracy of the combination of these tests has been studied (sensitivity and specificity $\geq 0.74$, positive likelihood ratio=3-5) ${ }^{19}$ Participants also had to have been pain-free for at least 3 months before the onset of the current shoulder episode. Participants were excluded if they presented one of the following conditions: (1) upper limb fracture; (2) previous neck or shoulder surgery; (3) shoulder pain reproduced during active neck movement; (4) frozen shoulder; (5) clinical signs of a full thickness RC tear (positive lag signs) ${ }^{2021}$; and (6) rheumatoid, inflammatory or neurological diseases.

\section{Design}

This single-blind (assessor), parallel-group RCT included three evaluation sessions over 6 weeks (baseline, week
2 and week 6) and two meetings with the treating physiotherapist. All participants took part in the baseline evaluation. They first completed a questionnaire on sociodemographic, symptomatology and comorbidity, as well as self-administered questionnaires that evaluate symptoms and functional limitations: Disabilities of the Arm, Shoulder and Hand (DASH), Western Ontario Rotator Cuff Index (WORC), and Brief Pain Inventory (BPI). Then, ultrasonographic (US) measurements of AHD, shoulder isometric strength and active ROM were conducted. Thereafter, participants were randomly assigned to one of two intervention groups, and then took part in their assigned intervention. The two intervention groups were group I: the experimental group that performed isometric exercises; and group II: the control group that received advice to rest from aggravating activities and were instructed to apply ice wraps. After the first 2 weeks, both groups then performed a graduated rehabilitation programme.

At weeks 2 and 6, questionnaires were readministered. US, strength and ROM measurements were only re-evaluated at week 2. To evaluate the effectiveness of blinding, the assessor completed a question related to her opinion of the allocation at week 2 .

\section{Sample size}

An a priori sample size was calculated based on the changes reported by the DASH scores for individuals with RC tendinopathy. ${ }^{17}$ According to sample size calculation $\left(G^{*}\right.$ Power 3.1.7, $\alpha=0.05$, effect size $=0.85$, power $[1-\beta]=0.80, \mathrm{SD}=13 \mathrm{DASH}$ points, clinically important difference $(\mathrm{CID})=11 \mathrm{DASH}$ points, $15 \%$ possible loss to follow-up), 22 participants per group were required.

\section{Randomisation/blinding}

A researcher not involved in data collection conducted randomisation prior to the initiation of the study, using a random number generator and a block design (block size of 4-6-8). Allocation was concealed in sealed opaque envelopes which were sequentially numbered. Randomisation was stratified to ensure balance of the treatment groups with respect to sex. Given that it is impossible to blind the treating physiotherapist and participants, a single-blind design was used. Participants, however, were unaware of the treatment provided to other participants. Participants were instructed not to reveal or discuss treatment with the evaluator. Blinded outcome assessment was carried out in person at baseline and 2 weeks, and via postal questionnaires at 6 weeks.

\section{Intervention}

Participants in both groups met the treating physiotherapist on two occasions (after the baseline and week 2 evaluations). The first meeting consisted of education regarding posture and positioning of the shoulder during daily activities to avoid additional stress on shoulder structures. This session also consisted of explanation and practice of the intervention to be carried out at home 
Table 1 Participants' baseline characteristics

\begin{tabular}{|c|c|c|}
\hline & $\begin{array}{l}\text { Cryotherapy } \\
\text { group }\end{array}$ & $\begin{array}{l}\text { Exercises } \\
\text { group }\end{array}$ \\
\hline Characteristics & $(n=23)$ & $(n=20)$ \\
\hline Age, years, $X \pm S D$ & $43 \pm 13^{*}$ & $33 \pm 7^{*}$ \\
\hline Gender, male (\%) & $11(48)$ & $13(65)$ \\
\hline Weight, kg, X \pm SD & $76 \pm 13$ & $74 \pm 13$ \\
\hline 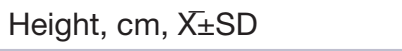 & $170 \pm 6$ & $175 \pm 9$ \\
\hline Days since the injury, $X \pm S D$ & $25 \pm 7$ & $27 \pm 9$ \\
\hline Dominance, right, \% & 83 & 90 \\
\hline Pain on dominant side, $\%$ & 52 & 65 \\
\hline \multicolumn{3}{|l|}{ Origin of injury, \% } \\
\hline Unknown & 4 & 0 \\
\hline Accident/Fall & 0 & 0 \\
\hline Repeated movement & 26 & 30 \\
\hline Sport & 39 & 55 \\
\hline Progressive & 31 & 15 \\
\hline \multicolumn{3}{|l|}{ Work status, \% } \\
\hline Full time & 78 & 65 \\
\hline Part-time & 9 & 10 \\
\hline Retired & 0 & 5 \\
\hline Student & 13 & 20 \\
\hline \multicolumn{3}{|c|}{ Physical work demands on the shoulder†, \% } \\
\hline I-sedentary & 0 & 0 \\
\hline II-light & 78 & 70 \\
\hline III-medium & 9 & 15 \\
\hline IV-high & 13 & 15 \\
\hline
\end{tabular}

*Denotes a statistically significant difference in mean score between the two groups $(p=0005)$.

†Average lifted load level to classify patients' occupation and its related level of physical demand on the shoulder. Sedentary was defined as general lifting activities not exceeding $6.8 \mathrm{~kg}$. Light was defined as lifting up 11.3-13.6 pounds. Moderate was defined as lifting up to 22.7-34.0pounds on a regular basis. High required lifting $34.0-45.4$ pounds on a regular basis.

$\mathrm{X}$, mean.

as per group allocation. Both groups were provided with written instructions describing their intervention and a treatment diary to record adherence.

In the gradual loading group, participants were provided with an isometric lateral rotation and abduction exercise programme to be carried out three times a day for 14 days. In a standing or sitting position, participants placed their affected arm by the side with the elbow flexed at $90^{\circ}$ and the thumb pointing upwards. The opposite hand was used to resist the lateral rotation and abduction. Participants were provided with a guide to the percentage of maximum force (ranging from $50 \%$ to $75 \%$ ), number of repetitions (3-4) and number of second holds (20-30 $\mathrm{s}$ ), which was progressed from day 1 to day 14 (online supplementary appendix 1). A maximum pain level of
$5 / 10$ was accepted when performing the contractions as long as it settled 10-15 min after the exercises.

In the rest and cryotherapy group, participants were asked to apply an ice wrap, secured within a towel, on their painful shoulder for 15 min three times per day. All participants were provided with a commercial ice wrap. They were also asked to avoid painful movements, working above shoulder level, repeated or sustained shoulder elevation movements, and lifting weights.

To avoid shoulder compensation during arm elevation and ankylosis, ${ }^{22}$ participants of both groups were instructed to carry out five repetitions of gentle, slow, pain-free active shoulder elevation movements in front of a mirror, in three planes (flexion, scaption and abduction). They were instructed to perform these movements at home, after each session of their respective programme.

At the second meeting, participants from both groups received the same strengthening exercise programme to be performed every day for the next 4 weeks. It included resisted isotonic shoulder external and internal rotation exercises using commercial elasticised rubber band. The exercises were progressed from $0^{\circ}$ abduction to $45^{\circ}$ and then $90^{\circ}$ abduction when the movements were easily executed and pain-free (online supplementary appendix 2).

\section{Outcome measures}

The primary outcome measure was function evaluated with the validated French-Canadian version of the DASH (intraclass correlation coefficient $(\mathrm{ICC})=0.93 \quad[0.87-$ 0.96]; minimal detectable change (MDC) $90 \%=11.4 \%$, $\mathrm{CID}=10.2$ points) ${ }^{23}$ It consists of 30 items addressing the level of difficulty in performing daily activities and severity of the symptoms. ${ }^{24} \mathrm{~A}$ score of 100 points indicates the most severe disability. ${ }^{25}$

The validated French-Canadian version of the WORC index $\quad(\mathrm{ICC}=0.96 \quad[0.92-0.98], \quad \mathrm{MDC} \quad 90 \%=12.3 \%$, $\mathrm{CID}=17.5 \%)^{23}$ was used to evaluate symptoms and functional limitations specific to RC disorders. It uses 21 questions, with $100 \mathrm{~mm}$ visual analogue scale responses, on pain and physical symptoms, sports recreation, and work, social and emotional functions. ${ }^{23} 26$ The Short Form of Brief Pain Inventory (ICC $>0.80)^{2728}$ was used to assess shoulder pain intensity. The BPI uses an 11-point numerical rating scale $(0-10)$ to evaluate pain interference over the last 24 hours. ${ }^{27} 28$

Ultrasound imaging was performed using Logiq E9 (GE Healthcare, Milwaukee, Wisconsin, USA) ultrasound scanner with a $7.5-12-\mathrm{MHz}$ linear array probe. AHD (measured at $0^{\circ}, 45^{\circ}$ and $60^{\circ}$ shoulder abduction) was measured using a standardised procedure, which has been previously demonstrated to be valid ${ }^{29}$ and reliable. ${ }^{30}$ The US measurement of AHD is defined as the tangential distance between the hyperechoic bony landmarks of the humeral head and the inferior edge of the acromion visible on the longitudinal sonogram. Measurement obtained represents the AHD at the anterior outlet of the subacromial space. 
Table 2 DASH, WORC, BPI, AHD, shoulder strength and ROM for cryotherapy and exercises groups at baseline and followup

\begin{tabular}{|c|c|c|c|c|}
\hline \multirow[b]{3}{*}{ Outcome measures } & \multirow[b]{3}{*}{ Week } & \multirow{2}{*}{$\begin{array}{l}\text { Cryotherapy group } \\
(n=23)\end{array}$} & \multirow{2}{*}{$\begin{array}{l}\text { Exercises group } \\
(n=20)\end{array}$} & \multirow{2}{*}{$\begin{array}{l}\text { Mean difference } \\
(95 \% \mathrm{Cl})\end{array}$} \\
\hline & & & & \\
\hline & & Mean score & Mean score & \\
\hline \multirow[t]{3}{*}{ DASH (0-100) } & 0 & $28.3(15.3)$ & $29.3(12.6)$ & $-1.0(-9.7$ to 7.7$)$ \\
\hline & 2 & $17.2(11.5)$ & $16.3(11.3)$ & 0.9 (-6.1 to 9.0$)$ \\
\hline & 6 & $12,6(12.5)$ & $16.3(12.1)$ & $-3.7(-11.3$ to 3.9$)$ \\
\hline \multirow[t]{3}{*}{ WORC (0-100) } & 0 & $57.9(19.6)$ & $55.7(21.0)$ & $2.2(-10.3$ to 14.8$)$ \\
\hline & 2 & $75.2(18.2)$ & $80.6(17.1)$ & $-5.4(-16.3$ to 5.5$)$ \\
\hline & 6 & $83.1(15.0)$ & $83.3(19.3)$ & $-0.2(-10.7$ to 10.4$)$ \\
\hline \multirow[t]{3}{*}{ BPI (0-10) } & 0 & $2.7(1.6)$ & $3.2(1.9)$ & $-0.5(-1.5$ to 0.6$)$ \\
\hline & 2 & $1.7(1.2)$ & $1.8(1.8)$ & $-0.1(-1.1$ to 0.8$)$ \\
\hline & 6 & $1.2(1.3)$ & $1.6(1.9)$ & $-0.4(-1.4$ to 0.6$)$ \\
\hline \multirow[t]{2}{*}{$\mathrm{AHD} 0^{\circ}, \mathrm{cm}$} & 0 & $1.1(0.1)$ & $1.0(0.2)$ & $0.1(-0.0$ to 0.2$)$ \\
\hline & 2 & $1.1(0.1)$ & $1.0(0.1)$ & 0.1 (-0.0 to 0.2$)$ \\
\hline \multirow[t]{2}{*}{ AHD $45^{\circ}, \mathrm{cm}$} & 0 & $0.8(0.2)$ & $0.8(0.2)$ & 0.0 (-1.0 to 0.2$)$ \\
\hline & 2 & $0.8(0.1)$ & $0.8(0.2)$ & $0.0(-1.0$ to 0.2$)$ \\
\hline \multirow[t]{2}{*}{$\mathrm{AHD} 60^{\circ}, \mathrm{cm}$} & 0 & $0.8(0.2)$ & $0.7(0.2)$ & 0.1 (-0.0 to 0.2$)$ \\
\hline & 2 & $0.8(0.2)$ & $0.7(0.2)$ & 0.1 (-0.0 to 0.2$)$ \\
\hline \multirow[t]{2}{*}{$\mathrm{ABD} 0^{\circ}$, strength, $\mathrm{kg}$} & 0 & $67.4(34.7)$ & $77.1(34.9)$ & $-9.7(-33.3$ to 14.0$)$ \\
\hline & 2 & $74.7(33.7)$ & $77.6(41.4)$ & $-2.9(-28.3$ to 22.6$)$ \\
\hline \multirow[t]{2}{*}{ ER $0^{\circ}$, strength, kg } & 0 & $23.5(10.8)$ & $25.8(9.3)$ & $-2.3(-9.2$ to 4.5$)$ \\
\hline & 2 & $24.8(10.8)$ & $28.0(9.6)$ & $-3.2(-10.1$ to 3.8$)$ \\
\hline \multirow[t]{2}{*}{ ROM flexion, $^{\circ}$} & 0 & $165.9(14.1)$ & $165.4(10.9)$ & 0.5 (-7.3 to 8.3$)$ \\
\hline & 2 & $166.7(7.9)$ & $167.5(9.0)$ & $-0.8(-6.6$ to 4.4$)$ \\
\hline \multirow[t]{2}{*}{ ROM ER $0^{\circ}, \circ$} & 0 & $68.3(15.9)$ & $71.7(10.6)$ & $-3.4(-11.9$ to 5.0$)$ \\
\hline & 2 & $68.4(15.9)$ & $70.5(12.5)$ & $-2.1(-11.4$ to 7.1$)$ \\
\hline \multirow[t]{2}{*}{ ROM ER $90^{\circ}, \circ$} & 0 & 90.7 (15.6) & $95.2(15.3)$ & $-4.5(-14.0$ to 5.1$)$ \\
\hline & 2 & $95.2(14.6)$ & $97.9(12.1)$ & $-2.7(-11.0$ to 5.7$)$ \\
\hline \multirow[t]{2}{*}{ ROM IR $90^{\circ},{ }^{\circ}$} & 0 & $70.1(17.2)$ & $74.3(17.0)$ & $-4.2(-14.8$ to 6.3$)$ \\
\hline & 2 & $68.7(14.1)$ & $72.4(17.6)$ & $-3.7(-13.4$ to 6.1$)$ \\
\hline
\end{tabular}

Data presented as mean (SD). Intention-to-treat analyses for DASH, WORC and BPI.

ABD, Abduction; AHD, acromiohumeral distance; BPI, Brief Pain Inventory, short version (0-10, $0=$ no pain); DASH, Disabilities of the Arm, Shoulder and Hand questionnaire 24 (0-100, $0=$ no disability); ER, external rotation of the shoulder; IR, internal rotation of the shoulder; ROM, range of motion; WORC, Western Ontario Rotator Cuff index (0-100, 100=no functional loss).

Shoulder abduction and shoulder external rotation maximum force were assessed in supine with the shoulder at $0^{\circ}$ flexion and the elbow at $90^{\circ}$ flexion, using a Medup handheld dynamometer (Atlas Medic, Quebec City, Canada). Shoulder ROM (flexion, external rotation at $0^{\circ}$ and $90^{\circ}$ abduction, shoulder internal rotation at $90^{\circ}$ abduction) was measured in supine using a digital inclinometer with reported intrarater reliability of ICC $=0.95-0.98 .^{31}$

\section{Data handling and statistical analyses}

Baseline demographic data were compared between groups using independent t-test and $\chi^{2}$. Intention-totreat (using last observation carried forward method for handling missing data) and per-protocol analyses were used for all outcomes.

For DASH, BPI and WORC, non-parametric repeated-measures analyses of variance (ANOVAs) for longitudinal data (nparLD, Package nparLD 2.1, R software, V.3.3.3) were used since distributions were normal at baseline and gamma at week 2 and week 6 (as most participants improved close to optimal values). ${ }^{32} \mathrm{~A}$ two-way (2-groups $\times$ 3-time) nparLD ANOVA was used to compare the intervention effects on DASH, BPI and WORC.

As far as assumptions were reached, the effects of interventions on AHD, ROM and isometric strength were 


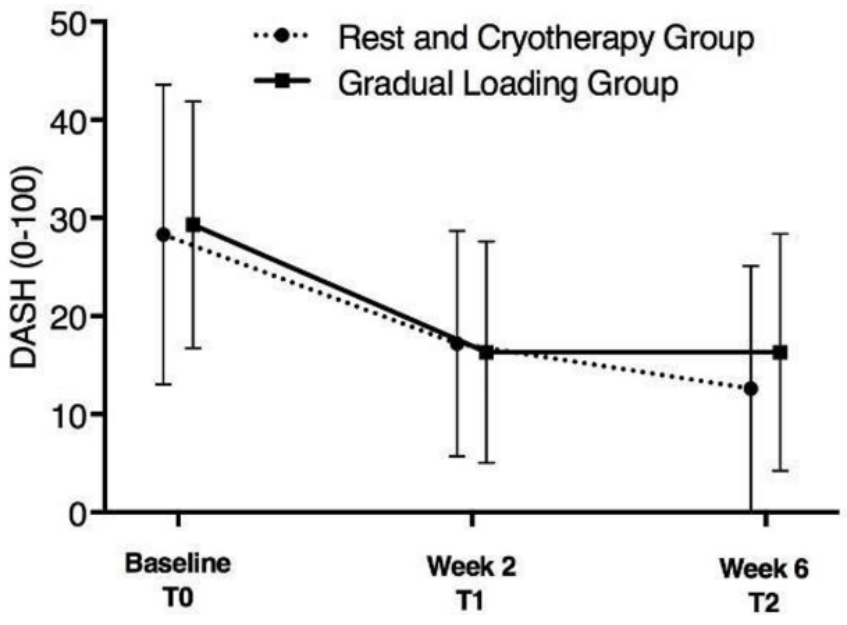

Figure 1 Mean score of DASH score at baseline, week 2 and week 6 of both groups. Error bars indicate SD. DASH, Disabilities of the Arm, Shoulder and Hand questionnaire (0-100, 0=no disability).

analysed with a three-way repeated-measures (groups $\times$ times $\times$ plane of movements) ANOVA (SPSS V.20). Effect sizes (Glass's $\Delta$ or $\eta^{2}$ ) were reported for all outcomes. The $\alpha$ criterion was set at $5 \%$ for all statistical analyses.

\section{RESULTS}

\section{Participants}

Between June 2016 and March 2018, 44 participants from 107 potential candidates were included (online supplementary appendix 3). All participants took part in the 2-week programme they were assigned to, but one participant was excluded at week 2 follow-up meeting due to a diagnosis of frozen shoulder (follow-up rate at week 2: $97.7 \%$ [cryotherapy group: 100\%; exercises group: 95\%]), while nine participants failed to return their follow-up questionnaires at week 6 (follow-up rate at week 6: $77.3 \%$ [cryotherapy group: $87.0 \%$; exercises

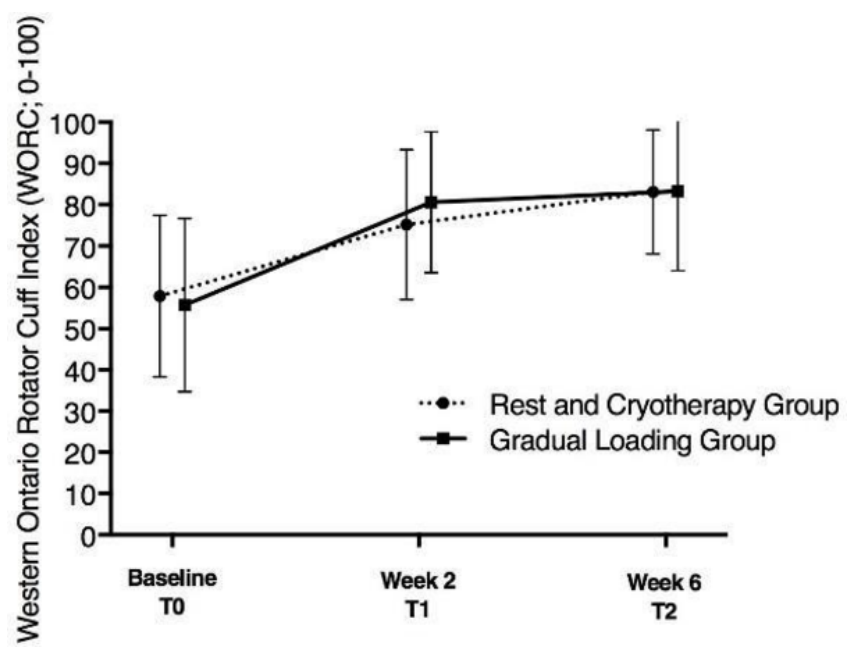

Figure 2 Mean score of WORC score at baseline, week 2 and week 6 of both groups. Error bars indicate SD. WORC, Western Ontario Rotator Cuff index (0-100, 100=no functional loss).

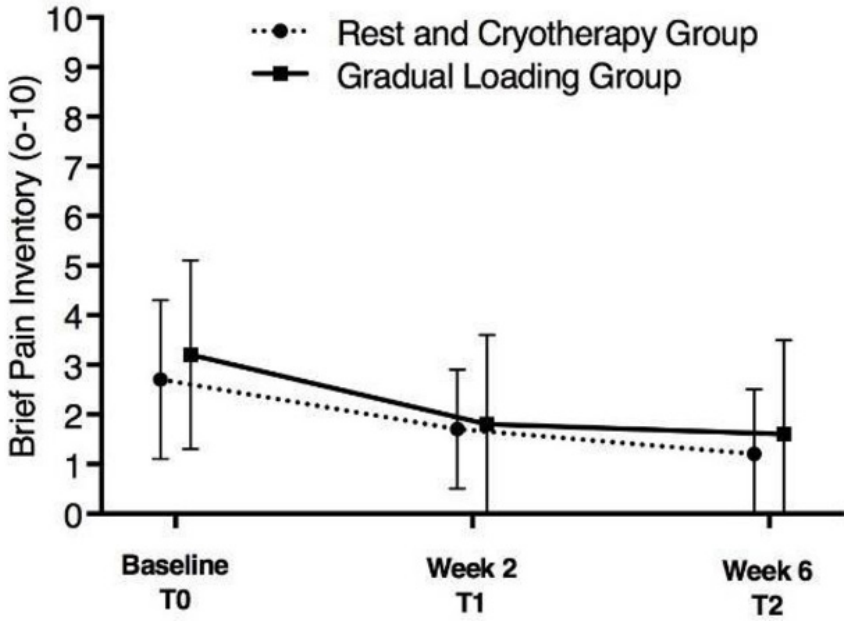

Figure 3 Mean score of Brief Pain Inventory score at baseline, week 2 and week 6 of both groups. Error bars indicate SD.

group: $70 \%$ ]). At week 2, self-declared compliance to the assigned programme was not significantly different between the groups (out of 42 sessions [ 3 sessions/day]: $\mathrm{p}=0.43$, mean of $33.6 \pm 5.3$ sessions of ice application for the cryotherapy group and mean of $31.9 \pm 6.2$ sessions of gradual reloading exercises for the exercises group). At week 6, self-declared compliance to the exercises programme that had to be done during weeks $3,4,5$ and 6 was not significantly different between the groups (out of 28 days: $p=0.076$, mean of $19.5 \pm 13.8$ days for the cryotherapy group and mean of $11.4 \pm 7.9$ days for the exercises group). No participant reported adverse effects. At week 6 , the assessor declared to be unaware of the group allocation of any participants. The two groups' baseline characteristics differed with regard to age, with the participants in the cryotherapy group being significantly older $(p=0.005$; table 1$)$. The use of age as a covariate was considered, but there was no correlation between age and DASH, WORC or BPI scores for all groups at all measurement times. Thus, age does not appear to be an influencing factor.

\section{Effect of early loading exercises and cryotherapy on pain and function}

Intention-to-treat and per-protocol analyses showed no significant group effect $(\mathrm{p}>0.05)$ and group $\times$ time interaction ( $\mathrm{p} \geq 0.12$; table 2, figures 1-3). However, both groups showed statistically significant improvement at weeks 2 and 6 in DASH, WORC and BPI scores compared with baseline $(\mathrm{p}<0.05$; table 3$)$, and DASH and WORC CIDs were achieved at week 2 (mean score difference from baseline [all groups]: 12.0 DASH points and 20.8 WORC points).

\section{Effect of early loading exercises and cryotherapy on US measurement, shoulder strength and ROM}

The analyses showed no group effect and no time effect on AHD, shoulder ROM and shoulder isometric strength 
Table 3 Mean scores difference of DASH, WORC, BPI, AHD, shoulder strength and ROM in relation to baseline values for cryotherapy and exercises group

\begin{tabular}{|c|c|c|c|c|}
\hline \multirow[b]{4}{*}{ Outcome measures } & \multirow[b]{4}{*}{ Week } & \multirow{2}{*}{$\begin{array}{l}\text { Cryotherapy group } \\
(n=23)\end{array}$} & \multirow{2}{*}{$\begin{array}{l}\text { Exercises group } \\
(n=20)\end{array}$} & \multirow{2}{*}{$\begin{array}{l}\text { Both groups } \\
(n=43)\end{array}$} \\
\hline & & & & \\
\hline & & $\begin{array}{l}\text { Mean score difference from } \\
\text { baseline }\end{array}$ & $\begin{array}{l}\text { Mean score difference from } \\
\text { baseline }\end{array}$ & $\begin{array}{l}\text { Mean score difference from } \\
\text { baseline }\end{array}$ \\
\hline & & $(95 \% \mathrm{Cl})$ & $(95 \% \mathrm{Cl})$ & $(95 \% \mathrm{Cl})$ \\
\hline \multirow[t]{2}{*}{ DASH (0-100) } & 2 & 11.1 (5.6 to 16.6$)$ & 13.1 (8.2 to 18.0$)$ & 12.0 (8.4 to 15.6$)$ \\
\hline & 6 & 15.7 (9.6 to 21.8$)$ & 13.1 (7.6 to 18.5$)$ & 14.5 (10.5 to18.5) \\
\hline \multirow{2}{*}{$\begin{array}{l}\text { Time effect } \\
\text { WORC (0-100) }\end{array}$} & 2 & 17.3 (9.5 to 25.0$)$ & 24.9 (14.7 to 35.2$)$ & 20.8 \\
\hline & 6 & 25.2 (16.1 to 34.3$)$ & 27.6 (15.9 to 39.3$)$ & 26.3 \\
\hline Time effect & & $\eta^{2}=0.603$ & $\eta^{2}=0.583$ & $\eta^{2}=0.587$ \\
\hline BPI (0-10) & 2 & 1.1 (0.3 to 1.8$)$ & 1.3 (0.8 to 1.9$)$ & 1.2 (0.7 to 1.6$)$ \\
\hline AHD $45^{\circ}, \mathrm{cm}$ & 2 & $0.0(-0.0$ to 0.1$)$ & $0.0(-0.0$ to 0.1$)$ & $0.0(-0.0$ to 0.1$)$ \\
\hline $\mathrm{AHD} 60^{\circ}, \mathrm{cm}$ & 2 & $0.0(-0.0$ to 0.1$)$ & $0.0(-0.1$ to 0.1$)$ & $0.0(-0.0$ to 0.1$)$ \\
\hline ABD $0^{\circ}$, strength, $\mathrm{kg}$ & 2 & $7.3(0.27$ to 14.4$)$ & $1.6(-7.6$ to 10.9$)$ & $4.7(-0.8$ to 10.2$)$ \\
\hline ER $0^{\circ}$, strength, kg & 2 & $1.4(-0.2$ to 2.9$)$ & $2.6(1.1$ to 4.1$)$ & 2.0 (0.9 to 3.0$)$ \\
\hline ROM flexion, ${ }^{\circ}$ & 2 & 0.7 (-3.5 to 4.9$)$ & $2.1(-3.6$ to 7.7$)$ & $1.3(-2.0$ to 4.7$)$ \\
\hline ROM ER $0^{\circ}, \circ$ & 2 & $0.1(-3.9$ to 4.1$)$ & $-1.2(-6.2$ to 3.8$)$ & $-0.5(-3.5$ to 2.5$)$ \\
\hline ROM ER $90^{\circ},{ }^{\circ}$ & 2 & $4.5(-2.8,11.9)$ & 2.7 (-2.5 to 7.9$)$ & $3.7(-0.8$ to 8.1$)$ \\
\hline ROM IR $90^{\circ}, \circ$ & 2 & $-1.4(-8.2$ to 5.4$)$ & $-1.9(-10.9$ to 7.0$)$ & $-1.7(-6.9$ to 3.6$)$ \\
\hline
\end{tabular}

Data presented as mean $(95 \% \mathrm{Cl})$. Intention-to-treat analyses for DASH, WORC and BPI.

ABD, Abduction; AHD, acromiohumeral distance; BPI, Brief Pain Inventory, short version (0-10, 0=no pain); DASH, Disabilities of the Arm, Shoulder and Hand questionnaire (0-100, $0=$ no disability); ER, external rotation of the shoulder; IR, internal rotation of the shoulder; ROM, range of motion; WORC, Western Ontario Rotator Cuff index (0-100, 100=no functional loss).

( $\mathrm{p} \geq 0.11$, tables 2 and 3 ), as well as no time $\times$ group interaction ( $\mathrm{p} \geq 0.18$; table 2 ).

\section{DISCUSSION}

The aim of this study was to compare a gradual reloading exercise programme with cryotherapy for acute RC tendinopathy. Our hypothesis that the early reloading exercises would be more effective in reducing pain and disability was not supported as both groups improved equally. Both groups showed statistically and clinically significant improvement in DASH, WORC and BPI scores at weeks 2 and 6.

It is known that shoulder pain can have an impact on the kinematics and the muscle activation pattern of the injured shoulder, ${ }^{33} 34$ which leads to altered movement patterns that can compromise recovery and lead to chronicity. Rehabilitation programmes that include controlled elevation movements to avoid compensation have been shown to be effective in improving shoulder control and in reducing pain and functional limitations in individuals with chronic RC tendinopathy. ${ }^{17} 2235$ Thus, the gentle and pain-free movements performed in front of a mirror by every participant may have been sufficient to improve shoulder control and to reduce pain during arm elevation, and so no additional benefits were provided by the presence of ice or isometric exercises. As no control group was included in the study, it is impossible to conclude on the effectiveness of the groups, and outcomes may be attributed to (1) mapping natural improvement, (2) placebo and (3) comparable beneficial treatment effects between the two groups, or a combination of these.

Tendons present a complex healing process that involves structural, cellular and vascular changes over time. ${ }^{13}$ During the 6 -week follow-up, the natural tendon healing process, which implies a gradual decrease in inflammatory and pain mediators, ${ }^{13}$ could explain the similar progression of the two groups regarding pain and function. The effect of ice or gradual exercises could be negligible compared with the time factor, which could explain the lack of difference between the two groups in the short term. The strength, ROM and AHD results, which did not improve over time for both groups, support this hypothesis.

A recent literature review ${ }^{36}$ suggested that an imbalanced adaptation between muscles and tendons in 
response to specific loading conditions might be responsible for tendon injuries. Muscles can improve in their strength and size under moderate loads, while tendons only seem to effectively be strengthened (increase stiffness) when high-magnitude tendon strain is applied (ie, $90 \%$ isometric maximum voluntary contraction). This imbalance leads to a higher tendon strain during maximal voluntary contraction and increases the mechanical demand on the tendon by the muscle contraction, which increases the risk of tendon injury. Thus, it is reasonable to believe that including gradual reloading exercises early in the rehabilitation that leads to high-magnitude tendon strain could be beneficial to address possible imbalances that could be responsible for the acute tendinopathy, which in turn would help prevent progression to chronicity. However, our protocol did not include such high reinforcement parameters, which would not have been enough to create a tendon adaptation. Future studies should include higher reinforcement parameters to observe the effect of early loading exercises.

The second objective of this study was to determine if these two interventions have different effects on shoulder strength, ROM and AHD. There was no significant difference between the two groups and there was no time effect. At baseline, both groups had AHD within the normal range, so there was no capacity or requirement for it to increase as expected.$^{37}$ Similarly, the mean ROM values at baseline were close to the normative values $^{38}$ and no ROM exercise was included in our interventions; therefore, it is not really surprising that ROM did not significantly change over time. Since 3-4 weeks are necessary to observe an increase in strength following resistance exercises, ${ }^{36}$ it also seems normal to observe no strength improvement within 2 weeks of treatment. Force measurements at 6-week follow-up would have made it easier to see if there is a variation in strength following these two interventions.

\section{Limitations}

There are some limitations to this study. First, there was no 'no treatment group' included. Therefore, resolution of symptoms due to the natural healing process cannot be excluded as the main reason of improvement. Future studies should measure tendon thickness alongside $\mathrm{AHD}$, as this is more likely to change in the acute stage of injury. ${ }^{39}$ Finally, there was a low follow-up rate at week 6 , especially in the exercises group, which means that the results could have been different had we achieved a better follow-up in the latter stage of the study.

\section{CONCLUSION}

Our results do not demonstrate any short-term differences between cryotherapy and a gradual reloading exercise programme in improving pain and function in the acute RC tendinopathy population. Considering the acute pain context, time could be a significant factor in reducing pain and functional disability, making negligible the effect of a reloading exercises or the use of ice in the short term.

\section{Author affiliations}

${ }^{1}$ Faculty of Medicine, Laval University, Quebec City, Canada

${ }^{2}$ Centre for Interdisciplinary Research in Rehabilitation and Social Integration, Quebec City, Canada

${ }^{3}$ School of Nursing and Midwifery, National University of Ireland Galway, Galway, Ireland

${ }^{4}$ Department of Clinical Therapies, Faculty of Education and Health Sciences, University of Limerick, Limerick, Ireland

${ }^{5}$ Health Research Institute, University of Limerick, Limerick, Ireland

${ }^{6}$ Department of Allied Health Professions and Midwifery, School of Health and Social

Work, University of Hertfordshire, Hatfield, UK

${ }^{7}$ Therapy Department, Central London Community Healthcare National Health Service Trust, London, UK

Contributors EB, KM, JSL and J-SR designed the study. EB, KM, JSL and J-SR created the study protocol. FD, EB and M-OD collected the data. FD and J-SR performed statistical analyses. FD and J-SR directed the publication. All authors contributed to writing and approved the final version of the manuscript.

Funding This research has been made possible by the awarding of a James $M$ Flaherty Research Scholarship from the Ireland Canada University Foundation, with the assistance of the Irish Government. J-SR was supported by a salary award from the CIHR and FRQ-S.

Competing interests None declared.

\section{Patient consent for publication Obtained.}

Ethics approval The sectorial rehabilitation and social integration research ethics committee of the CIUSSS-CN approved this study.

Provenance and peer review Not commissioned; internally peer reviewed.

Open access This is an open access article distributed in accordance with the Creative Commons Attribution Non Commercial (CC BY-NC 4.0) license, which permits others to distribute, remix, adapt, build upon this work non-commercially, and license their derivative works on different terms, provided the original work is properly cited, appropriate credit is given, any changes made indicated, and the use is non-commercial. See: http://creativecommons.org/licenses/by-nc/4.0/

\section{REFERENCES}

1. Kooijman M, Swinkels I, Dijk Cvan, et al. Patients with shoulder syndromes in general and physiotherapy practice: an observational study. BMC Musculoskelet Disord 2013;14:128.

2. Redondo-Alonso L, Chamorro-Moriana G, Jiménez-Rejano JJ, et al. Relationship between chronic pathologies of the supraspinatus tendon and the long head of the biceps tendon: systematic review. BMC Musculoskeletal Disorders 2014;15.

3. Tekavec E, Jöud A, Rittner R, et al. Population-based consultation patterns in patients with shoulder pain diagnoses. BMC Musculoskeletal Disorders 2012;13:238

4. Whittle S, Buchbinder R. In the clinic. Rotator cuff disease. Ann Intern Med 2015;162:ITC1-15.

5. McCreesh KM, Crotty JM, Lewis JS. Acromiohumeral distance measurement in rotator cuff tendinopathy: is there a reliable, clinically applicable method? A systematic review. British Journal of Sports Medicine 2015;49:298-305.

6. Thornton GM, Shao X, Chung M, et al. Changes in mechanical loading lead to tendonspecific alterations in MMP and TIMP expression: influence of stress deprivation and intermittent cyclic hydrostatic compression on rat supraspinatus and Achilles tendons. British Journal of Sports Medicine 2010;44:698-703.

7. Seitz AL, McClure PW, Finucane S, et al. Mechanisms of rotator cuff tendinopathy: intrinsic, extrinsic, or both? Clinical Biomechanics 2011;26:1-12.

8. Cook JL, Purdam CR. Is tendon pathology a continuum? A pathology model to explain the clinical presentation of load-induced tendinopathy. British Journal of Sports Medicine 2009;43:409-16.

9. Lewis JS. Rotator cuff tendinopathy: a model for the continuum of pathology and related management. British Journal of Sports Medicine 2010;44:918-23.

10. Lake SP, Miller KS, Elliott DM, et al. Effect of fiber distribution and realignment on the nonlinear and inhomogeneous mechanical 
properties of human supraspinatus tendon under longitudinal tensile loading. J Orthop Res 2009;27:1596-602.

11. Tashjian RZ, Farnham JM, Albright FS, et al. Evidence for an inherited predisposition contributing to the risk for rotator cuff disease. J Bone Joint Surg Am 2009;91:1136-42.

12. Rechardt M, Shiri R, Karppinen J, et al. Lifestyle and metabolic factors in relation to shoulder pain and rotator cuff tendinitis: a population-based study. BMC Musculoskeletal Disorders 2010;11:165

13. Longo UG, Berton A, Khan WS, et al. Histopathology of rotator cuff tears. Sports Medicine and Arthroscopy Review 2011;19:227-36.

14. Murphy R, Kliskey K, Wheway K, et al. Short term tissue response to current treatments for rotator cuff tendinopathy. International Journal Of Experimental Pathology 2013;94:A9-A10.

15. Kuijpers T, van Tulder MW, van der Heijden GJMG, et al. Costs of shoulder pain in primary care consulters: a prospective cohort study in The Netherlands. BMC Musculoskelet Disord 2006;7:83.

16. Michener L, Subasi Yesilyaprak S, Seitz A, et al. Supraspinatus tendon and subacromial space parameters measured on ultrasonographic imaging in subacromial impingement syndrome. Knee Surgery, Sports Traumatology. Arthroscopy 2015;23:363-9.

17. Savoie A, Mercier C, Desmeules F, et al. Effects of a movement training oriented rehabilitation program on symptoms, functional limitations and acromiohumeral distance in individuals with subacromial pain syndrome. Manual Therapy 2015;20:703-8.

18. Parle PJ, Riddiford-Harland DL, Howitt CD, et al. Acute rotator cuff tendinopathy: does ice, low load isometric exercise, or a combination of the two produce an analgaesic effect? British Journal of Sports Medicine 2017;51:208-9.

19. Michener LA, Walsworth MK, Doukas WC, et al. Reliability and diagnostic accuracy of 5 physical examination tests and combination of tests for subacromial impingement. Arch Phys Med Rehabil 2009;90:1898-903.

20. Hertel R, Ballmer FT, Lombert SM, et al. Lag signs in the diagnosis of rotator cuff rupture. J Shoulder Elbow Surg 1996;5:307-13.

21. Miller CA, Forrester GA, Lewis JS. The validity of the lag signs in diagnosing full-thickness tears of the rotator cuff: a preliminary investigation. Arch Phys Med Rehabil 2008;89:1162-8.

22. Roy J-S, Moffet $\mathrm{H}$, Hébert LJ, et al. Effect of motor control and strengthening exercises on shoulder function in persons with impingement syndrome: a single-subject study design. Manual Therapy 2009;14:180-8.

23. St-Pierre C, Dionne CE, Desmeules F, et al. Reliability, validity, and responsiveness of a Canadian French adaptation of the Western Ontario Rotator Cuff (WORC) index. J Hand Ther 2015;28:292-9.

24. Durand M-J, Vachon B, Hong QN, et al. The cross-cultural adaptation of the DASH questionnaire in Canadian French. $J$ Hand Ther 2005;18:34-9.

25. Roy JS, MacDermid JC, Woodhouse LJ. Measuring shoulder function: a systematic review of four questionnaires. Arthritis Rheum 2009;61:623-32.
26. Kirkley A, Alvarez C, Griffin S. The development and evaluation of a disease-specific quality-of-life questionnaire for disorders of the rotator cuff: the western ontario rotator cuff index. Clin J Sport Med 2003;13:84-92.

27. Tan G, Jensen MP, Thornby Jl, et al. Validation of the brief pain inventory for chronic nonmalignant pain. J Pain 2004;5:133-7.

28. Tatham B, Smith J, Cheifetz O, et al. The efficacy of exercise therapy in reducing shoulder pain related to breast cancer: a systematic review. Physiother Can 2013:65:321-30.

29. McCreesh K, Adusumilli P, Evans T, et al. Validation of ultrasound measurement of the subacromial space using a novel shoulder phantom model. Ultrasound in Medicine \& Biology 2014;40:1729-33.

30. McCreesh KM, Anjum S, Crotty JM, et al. Ultrasound measures of supraspinatus tendon thickness and acromiohumeral distance in rotator cuff tendinopathy are reliable. Journal of Clinical Ultrasound 2016:44:159-66.

31. Kolber MJ, Hanney WJ. The reliability and concurrent validity of shoulder mobility measurements using a digital inclinometer and goniometer: a technical report. Int J Sports Phys Ther 2012;7:306-13.

32. Noguchi K, Gel YR, Brunner E, et al. nparLD: An $R$ Software Package for the Nonparametric Analysis of Longitudinal Data in Factorial Experiments. J Stat Softw 2012;50.

33. Ludewig P, Cook TM. Alterations in shoulder kinematics and associated muscle activity in people with symptoms of shoulder impingement. Physical Therapy 2000;80:276-91.

34. Roy J-S, Moffet H, McFadyen BJ. Upper limb motor strategies in persons with and without shoulder impingement syndrome across different speeds of movement. Clinical Biomechanics 2008;23:1227-36.

35. Worsley P, Warner M, Mottram S, et al. Motor control retraining exercises for shoulder impingement: effects on function, muscle activation, and biomechanics in young adults. J Shoulder Elbow Surg 2013;22:e11-9.

36. Mersmann F, Bohm S, Arampatzis A. Imbalances in the development of muscle and tendon as risk factor for tendinopathies in youth athletes: a review of current evidence and concepts of prevention. Front Physiol 2017;8

37. Mccreesh K, Donnelly A, Lewis J. Increased supraspinatus tendon thickness following fatigue loading in rotator cuff tendinopathy. Manual Therapy 2016;25:e41

38. Vairo GL, Duffey ML, Owens BD, et al. Clinical descriptive measures of shoulder range of motion for a healthy, young and physically active cohort. Sports Med Arthrosc Rehabil Ther Technol 2012;4:33.

39. McCreesh KM, Purtill H, Donnelly AE, et al. Increased supraspinatus tendon thickness following fatigue loading in rotator cuff tendinopathy: potential implications for exercise therapy. BMJ Open Sport Exerc Med 2017;3:e000279. 